\title{
Models for VLTI fringe sensor units: FINITO and PRIMA FSUs
}

\author{
Donata Bonino*, Mario Gai, Leonardo Corcione, Giuseppe Massone \\ Istituto Nazionale di AstroFisica - Osservatorio Astronomico di Torino \\ Via Osservatorio 20, 10025 Pino Torinese (TO) - Italy
}

\begin{abstract}
The VLTI system foresees two generations of fringe sensor: FINITO and PRIMA FSUs. The former is dedicated to H band; it controls the internal OPD with a temporal modulation with an external reference OPD. The latter, working with the $\mathrm{ABCD}$ model and in $\mathrm{K}$ band, is based on the introduction of known phase offsets for the interferometric signal (spatial phase modulation) and on the measurement of the corresponding combined power. Simulation models for both FSUs are developed with Matlab. Instrumental parameters, i.e. phase, transmission, visibility, are tabulated for ease of maintenance and to speed execution time. For the use of siderostats, due to fast turbulence, the need for intensity calibration arises. Assuming slow intensity variations with respect to phase variations, different algorithms can apply, yielding to numerical control of perturbations as a function of model parameters.
\end{abstract}

Keywords: VLTI, Fringe tracking, simulation, calibration, tolerances.

\section{INTRODUCTION}

The INAF - OATo have undertaken a collaboration with the European Southern Observatory (ESO) for the feasibility study, performance predictions and manufacturing for FINITO (Fringe-tracking Instrument of NIce and TOrino), a Fringe Sensor Unit (FSU) for the Very Large Telescope Interferometer ${ }^{1}$ (VLTI) located at Cerro Paranal, Chile. Our team is also involved in the development of the PRIMA (Phase Referenced Imaging and Micro-arcsecond Astrometry) FSU. The instrumental concept for both FSUs is described in this Conference ${ }^{2}$.

Whilst the operating concepts are different (respectively temporal and spatial modulation, $\mathrm{H}$ and $\mathrm{K}$ band), they are both based on the same description of polychromatic interferogram (see. Eq. 6). The aim of this paper is to present an outline of the algorithms for both FSUs.

An implementation of the outputs of an ideal FSU in a narrow spectral band around $\lambda_{0}$ is given by the ABCD model, in which two telescope beams generate four interferometric signals (respectively labelled A, B, C and D), built with phase difference of multiples of $\pi / 4$; most parameters are factored out as effective values, removing the integral over the bandwidth:

$$
A=\frac{I}{2}\left[1+V \sin \frac{2 \pi}{\lambda_{0}} x\right], B=\frac{I}{2}\left[1+V \cos \frac{2 \pi}{\lambda_{0}} x\right], C=\frac{I}{2}\left[1-V \sin \frac{2 \pi}{\lambda_{0}} x\right], D=\frac{I}{2}\left[1-V \cos \frac{2 \pi}{\lambda_{0}} x\right]
$$

Here, simple analytical relations among the measured signals provide the desired estimate of input parameters:

detected signal of individual telescope beam:

square visibility:

and OPD:

*bonino@to.astro.it, http://www.to.astro.it

$$
I=(A+B+C+D) / 2,
$$


In this ideal model the signals are periodic and what is measured is the OPD modulo the fringe period $\lambda_{0}$, i.e. the dynamic range of measurement is a single fringe period $\lambda_{0} / 2$ around one arbitrary reference position within the interferogram envelope. This description is not sufficiently accurate for the algorithm of a realistic instrument, but is useful as a starting point for more detailed implementation. The detailed instrument model must take into account the spectral distribution of source emission, phase, transmission, visibility, detector response, etc. Each parameter in the model has to be carefully calibrated to a precision defined by the sensitivity analysis, with dedicated procedures. Below we show some excerpts of the algorithms for FINITO (section 2) and for PRIMA FSU (section 3).

\section{FINITO}

FINITO is a fringe sensor working in the $\mathrm{H}$ band $(1.5-1.8 \mu \mathrm{m})$ which realizes an afocal pair-wise combination of two or three telescope beams at time. The interferometer stabilization is made possible by the measurement of the optical path difference (OPD) variation due to the atmospheric turbulence. The operational principles are explained in this Conference $^{2}$. Below (section 2.1) we derive a simplified model of the output interferogram which is used for the construction of the cophasing and coherencing algorithms. Moreover, during the development unexpected noise sources, characterized by higher frequency than expected, were detected, on both astronomical flux and on metrology beam intensities. In order to provide accurate modulation of the internal optical path, the need for metrology intensity monitoring and normalization was identified. Below (section 2.2) we show some of the possible approaches.

\subsection{Algorithm concept}

In FINITO, a relevant portion of the polychromatic interferogram is temporally modulated in order to explore the interferometric pattern, modeled as a modulated sinusoidal curve. The modulation term is strictly dependent from wavelengths. In the ideal case, the filter passband function $w(\lambda)$ is rectangular, depending on the effective wavelength $\lambda_{0}$ and on the bandwith $\Delta \lambda$; therefore the interferogram modulation function can be described as an integration over the full spectral bandwidth:

$$
m(x)=\int_{\lambda_{1}}^{\lambda_{2}} w(\lambda) \cos \left(2 \pi \frac{x}{\lambda}\right) d \lambda
$$

The interferogram is formed by two complementary waves, the constructive $(+)$ and the destructive $(-)$ ones. The former reaches its maximum when the optical path difference (OPD) is nulled, while the latter is in phase opposition with a minimum in the same abscissa. If we indicate with $F$ the constructive wave and with $G$ the destructive one, the interferogram is described as:

$$
F(x) ; G(x)=\frac{1}{2}\left\{I_{1}+I_{2} \pm 2 V \sqrt{I_{1} I_{2}} m(x)\right\}=\frac{1}{2}\left\{I_{1}+I_{2} \pm 2 V \sqrt{I_{1} I_{2}} \sum_{\lambda} w(\lambda) \cos \left(2 \pi \frac{x}{\lambda}\right)\right\}
$$

where $I_{1}$ and $I_{2}$ are the beam intensities and $V$ the visibility; the integral turns into a summation over the wavelength range for ease of implementation.

The modulation range must be smaller than the first lobe of the interferometric envelope, which is twice the coherence length, i.e. $L_{E}=2 \lambda^{2} / \Delta \lambda$, to avoid the lateral sections where the amplitude vanishes, but sufficient to avoid fringe jumps, i.e. rapid turbulence that cause OPD to change of more than $\lambda$.

In the algorithms, the OPD scan is performed applying a triangular optical path (OP) offset $x$ with a known fluctuation phase: 


$$
F(x) / G(x)=\frac{1}{2}\left\{I_{1}+I_{2} \pm 2 V \sqrt{I_{1} I_{2}} \sum_{\lambda} w(\lambda) \cos \left(2 \pi \frac{x+\text { phase }}{\lambda}\right)\right\}
$$

Beam intensities can be calibrated by the FSU using the photometric outputs; the visibility also requires the interferometric signals. In our model they are known quantities, i.e. estimated and updated at a lower frequency.

The $F$ and $G$ measurements $(y(t))$ are used for real time estimate of the OPD, taking into account that samples have a known phase offset, using either a standard demodulation procedure:

$$
\left\{\begin{array}{lll}
\int_{x} y(t) * \sin (2 \pi x) d x=1 / 2 \int_{x} \sin (2 \pi x+p h)-\sin (p h) d x & \Rightarrow & -1 / 2 \sin (p h) \\
\int_{x}^{x} y(t) * \cos (2 \pi x) d x=1 / 2 \int_{x} \sin (2 \pi x+p h)+\cos (p h) d x & \Rightarrow & 1 / 2 \cos (p h)
\end{array}\right.
$$

or a variation of the $\mathrm{ABCD}$ method.

This defines the cophasing algorithm, operating on a short segment of the interferogram.

The coherencing algorithm uses the same demodulation approach (eq. 8), but on longer time scale, in order to span a significant fraction of the full OPD range, to identify fringe jumps and allow for correction.

\subsection{Intensity monitoring on metrology channels}

This section is aimed to investigate the worst case of simultaneous variation on the same time scale of both phase and intensity. As an example, the metrology system is explored. In order to maintain the modulation law against fluctuation on the laser source and of the environment, the metrology makes a periodic scan and correction of the internal OPD. Metrology channels for one combination beams can be ideally represented as two sinusoidal waves in quadrature:

$$
s 1=A 1 \cdot I \cdot[1+V \sin \varphi] ; \quad s 2=A 2 \cdot I \cdot[1+V \cos \varphi]
$$

In our model, the coupling factor $A 1, A 2$ of the photodiodes monitoring the metrology source (a laser beam at $\lambda=1310 \mathrm{~nm}$ ) and the visibility $V$ are considered as known, while the intensity $I$ and the phase $\varphi$ are assumed to have independent variations on the same time scale. This allows use of an iterative procedure, able to correct progressively both parameters. We decompose intensity $I$ in the sum of a nominal intensity $I_{0}$ and a variation $\delta I_{0}$ :

$$
I=I_{0}+\delta I \text {. }
$$

We consider the signals normalised with respect to $A 1, A 2$ :

$$
n 1=I \cdot[1+V \sin \varphi] \quad n 2=I \cdot[1+V \cos \varphi]
$$

and the pure modulation component:

$$
p 1=\frac{s 1}{A 1}-I_{0}=\delta I_{0}+\left(I_{0}+\delta I_{0}\right) V \sin \varphi ; \quad p 2=\frac{s 2}{A 2}-I_{0}=\delta I_{0}+\left(I_{0}+\delta I_{0}\right) V \cos \varphi
$$

A first approximation for the phase can be done using the derived signals: $\quad \varphi_{\text {est }}=\arctan \frac{p 1}{p 2}$

A possible approach is the minimisation of an error function with a least square method.

The signals $p_{i}, i=1,2$, (see eq. (11)), are compared with two sinusoidal templates $t_{i}$ :

$$
\begin{array}{ccc}
p 1=\delta I_{0}+\left(I_{0}+\delta I_{0}\right) V \sin \varphi & \rightarrow & t 1=I_{0} V \sin \varphi_{\text {est }} \\
p 2=\delta I_{0}+\left(I_{0}+\delta I_{0}\right) V \cos \varphi & \rightarrow & t 2=I_{0} V \cos \varphi_{\text {est }}
\end{array}
$$


and the minimum of the error function $\left(p_{i}-t_{i}\right)^{2}, i=1,2$ is found by searching the zero position of its derivative versus $\varphi$, evaluated according to eq. (12).

$$
D(\varphi)=\sum_{i}\left(p_{i}(\varphi)-t_{i}(\varphi)\right)^{2}, \quad \frac{\partial D}{\partial I_{0}}=0 \Rightarrow I_{1}(\varphi)=\frac{p 1(\varphi) \cdot \sin \varphi_{e s t}+p 2(\varphi) \cdot \cos \varphi_{e s t}}{V}
$$

The procedure allows an iterative process for estimate of phase and intensity:

$$
\begin{gathered}
p 1, i=n 1-I_{i-1}, \quad p 2, i=n 2-I_{i-1}, \quad \varphi_{e s t, i}=\arctan \frac{p 1, i}{p 2, i}, \\
I_{i}(\varphi)=\frac{p 1, i(\varphi) \cdot \sin \varphi_{e s t, i}+p 2, i(\varphi) \cdot \cos \varphi_{\text {est }, i}}{V}
\end{gathered}
$$

In the simulation showed below, some parameters have a common fixed value and are summarised in this table:

\begin{tabular}{|l|c|l|c|}
\hline Coupling factor $A 1$ & 0.87 & Coupling factor $A 2$ & 0.85 \\
\hline Visibility $V$ & 0.9 & Nominal intensity $I_{0}$ & 12.34 \\
\hline Phase & {$[-\pi, \pi]$} & Intensity discrepancy & constant: $-2 \%$ of $I_{0}$ \\
\hline Number of phase steps & 1000 & Noise on phase & Suppressed \\
\hline
\end{tabular}

Hereafter, phase units are radians, while intensity is in arbitrary units. At the laser wavelength $(\lambda=1310 \mathrm{~nm})$, the relation between radians $x_{r}$ and nanometers $x_{m}$ is set by $x_{m}=\frac{1310 \mathrm{~nm}}{2 \pi} x_{r}$, so $1 \mathrm{rad} \approx 208.5 \mathrm{~nm}$.

The following pictures show the difference between estimated and nominal phase for two iteration steps (fig. 1a), and the estimated recursive intensity versus nominal intensity, again for two steps (fig. 2a). After two recursive steps there is no additional improvement on phase; intensity estimate saturates at the first iteration. The mean value of the discrepancy between nominal and estimated values at the second iteration is 12.092 with RMS 0.278 for the intensity, $9.9 \cdot 10^{-5}$ radians with RMS 0.018 radians for the phase.

The result is a reasonable approximation, but affected by significant systematic errors, in this implementation.

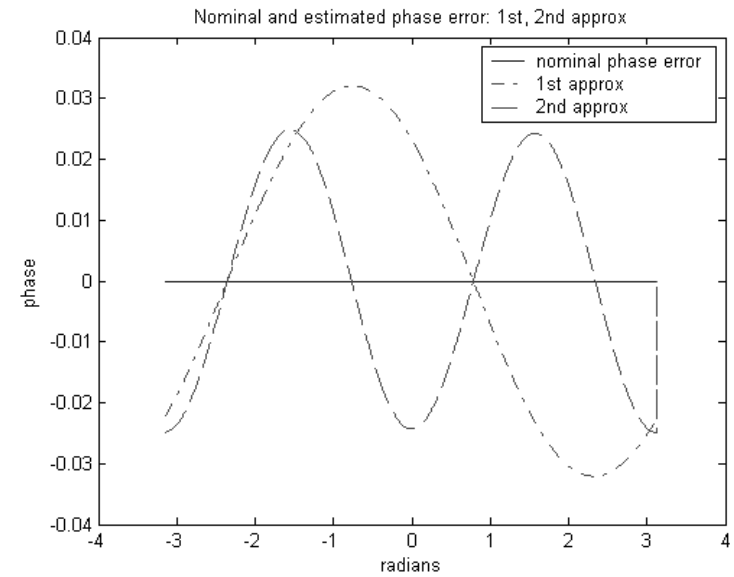

Fig. 1a: Nominal (solid line) and estimated (dash-dot: first approximation; dashed: second approximation) phase error

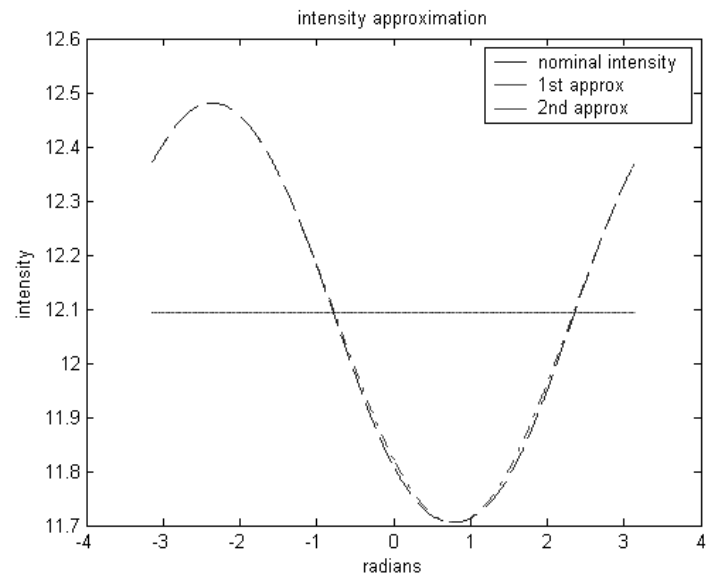

Fig. 1b: nominal (solid line) versus approximated (dash-dot, dashed lines, superposed: $1^{\text {st }}$ and $2^{\text {nd }}$ approximation) intensities 
Another approach is based on fitting an error function using templates for both $p 1$ and $p 2$ with variations on the intensity:

$$
t_{1}(\varphi, s f)=s f \cdot I_{0} \cdot V \cdot \sin (\varphi) \quad t_{2}(\varphi, s f)=s f \cdot I_{0} \cdot V \cdot \cos (\varphi)
$$

where $s f$ is an intensity scaling factor. This leads to the definition of an error function:

$$
e(\varphi, s f)=\left(p 1(\varphi)-t_{1}(\varphi, s f)\right)^{2}+\left(p 2(\varphi)-t_{2}(\varphi, s f)\right)^{2}
$$

For a set of different scaling factors, and for each punctual phase $\varphi$, we can find the quadratic polynomial fitting the set of points $[e(\varphi, s f)]$ and search its minimum. This gives the scaling factor $s f$ nearest to the noisy one for each $\varphi$; the estimated intensity is:

$$
I_{1}=s f \cdot I_{0} \text {. }
$$

Subsequent phase estimates might be achieved by setting:

$$
p 1, i=n 1-I_{i-1} \quad \quad p 2, i=n 2-I_{i-1}, \quad \varphi_{\text {est }, i}=\arctan \frac{p 1, i}{p 2, i} .
$$

However, as in the previous case, the method saturates at the first iteration, with comparable results.

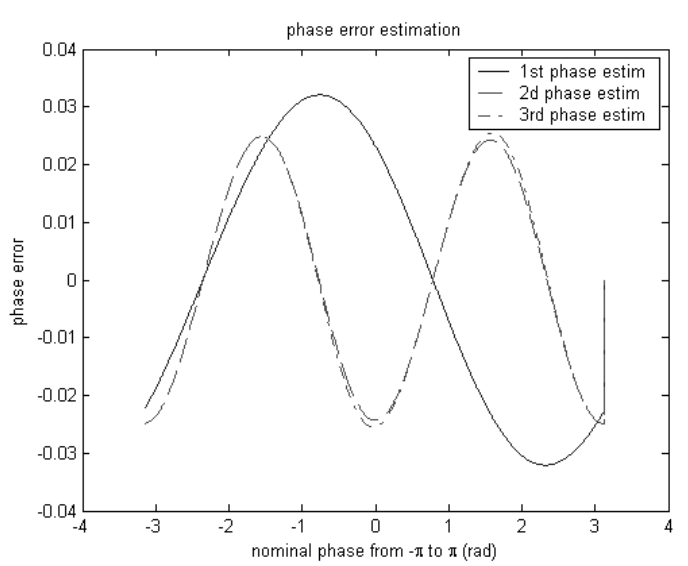

Fig. 2a: Subsequent phase estimates; solid line: first; dashed: second; dash-dot: third approximation

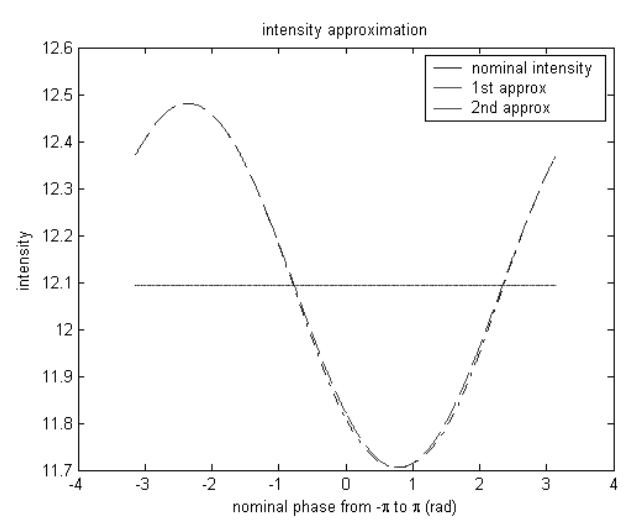

Fig. 2b: nominal intensity (solid line) vs. approximations (dash-dot, dashed lines, superposed: $1^{\text {st }}$ and $2^{\text {nd }}$ iteration)

Assuming small variation for the visibility, an expression for $\delta I$ can be find starting from the $n_{i}$ signals (eq. 10), evaluated with the $\varphi_{\text {est }}$ of equation (12):

$$
n 1^{2}+n 2^{2}=\left(I 0^{2}+2 I 0 \cdot \delta I+\delta I^{2}\right) \cdot\left[2+V^{2}+2 V\left(\sin \varphi_{e s t}+\cos \varphi_{e s t}\right)\right]
$$

If we truncate it at first order on $\frac{\delta I}{I 0}$ :

$$
\frac{n 1^{2}+n 2^{2}}{I 0^{2}} \cong 2+V^{2}+2 V\left(\sin \varphi_{e s t}+\cos \varphi_{\text {est }}\right)+2 \frac{\delta I}{I 0}\left[2+V^{2}+2 V\left(\sin \varphi_{\text {est }}+\cos \varphi_{\text {est }}\right)\right]
$$


we have:

$$
\delta I \cong \frac{I 0}{2}\left[\frac{n 1^{2}+n 2^{2}}{I 0^{2}\left[2+V^{2}+2 V\left(\sin \varphi_{\text {est }}+\cos \varphi_{\text {est }}\right)\right]^{-1}}\right]
$$

and the related intensity expression:

$$
\begin{aligned}
& I_{1} \cong I_{0}+\delta I \\
& 2+V^{2}+2 V(\sin \varphi+\cos \varphi)>0
\end{aligned}
$$

eq. 22 is always resolved.

Figure 3a and 3b show the comparison between the estimated phase, respectively intensity, versus the nominal one; it is evident a sinusoidal noise due to use of the estimated phase (12). The mean value of the discrepancy between nominal and estimated values at the second iteration is 12.188 with RMS 0.089 for the intensity, $8.8 \cdot 10^{-5}$ radians with RMS 0.016 radians for the phase.

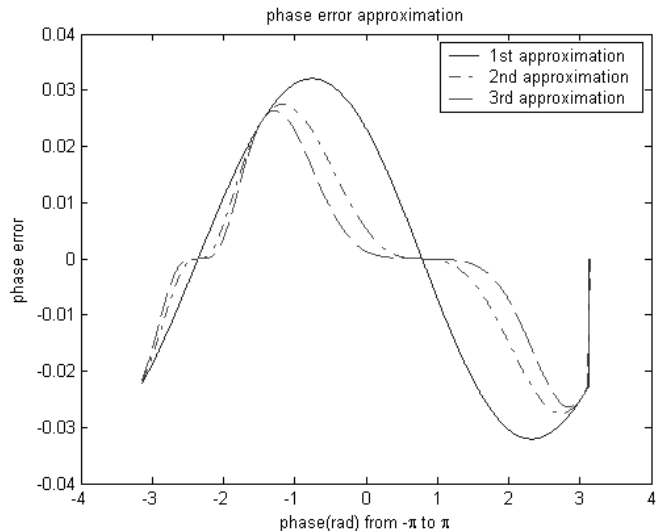

Fig. 3a: Subsequent phase estimates; solid line: first; dashdot: second; dashed: third approximation

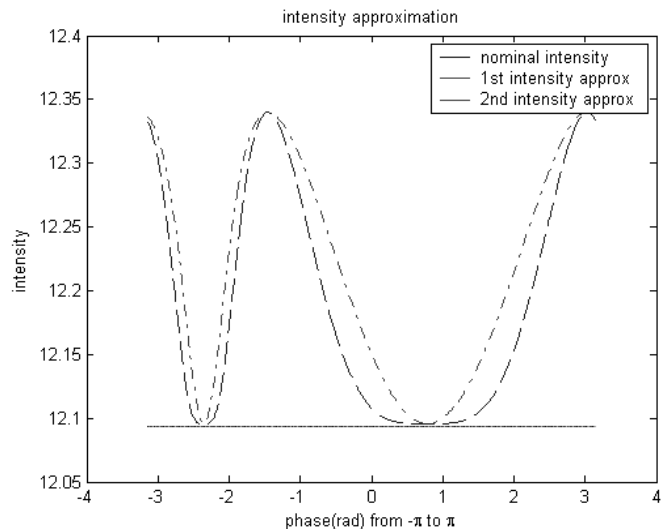

Fig. 3b: nominal intensity (solid line) vs. approximations (dash-dot, dashed lines: $1^{\text {st }}$ and $2^{\text {nd }}$ iteration)

Here the convergence is better than in previous cases, since there is significant improvement in subsequent iterations. In particular, over most of the range, the procedure appears to converge, with different number of iterations depending on the position. The technique might be acceptable, but there are two positions (approximately $\pi$ and $-\pi / 2$ ) in which the approximation error remains fairly high, i.e. the estimate sensitivity is low.

In most realistic cases, intensity variations are slower than the modulation, i.e. the phase estimate rate. This means a relaxed time constraint for the intensity estimation. However, this provides better operating conditions for the above procedure, since the OPD variation explores the full period, reducing the effect of the low sensitivity regions.

\section{PRIMA}

PRIMA FSU is a "fringe sensor", i.e. an interferometric instrument working in the K wavelength band $(2.0 \div 2.5 \mu \mathrm{m})$, with the possibility to be upgraded to work also in the $\mathrm{H}$ wavelength band $(1.48 \div 1.79 \mu \mathrm{m})$. Given two astronomical beams from a celestial source, collected by two different telescope apertures, the FSU superposes them to obtain an interferometric pattern from which it provides measurements of the optical path difference (OPD), the group delay (GD) and the amplitude of the fringes.

The operating principle of PRIMA FSU (explained in this Conference ${ }^{2}$ ) is different from FINITO one. For PRIMA, the OPD values are obtained with a spatial modulation of the optical path length of the two astronomical beams by mean of four known phase offsets, and by detecting the intensity of the combined beams in correspondence of these offsets. The GD is a measure of the dependence of the OPD from the wavelength, it is obtained by repeating the OPD measurements in three different $\mathrm{K}$ sub-bands. References to models and algorithms for phase and GD estimation in a fringe sensor can be found in literature ${ }^{2,3,4}$. PRIMA FSU is a good approximation of the ABCD model. 
From a realistic instrument description, dependent on spectral distribution of the source, the system characteristic, the environmental condition, a direct analytical solution is not immediate. It is possible to use a simplified model, similar to $\mathrm{ABCD}$, using suitable effective values for the parameters:

$$
y_{k} \cong \rho_{k} \cdot I_{k} \cdot\left[1+\eta_{k}^{P} \cdot \eta_{k}^{C} \cdot \eta_{k}^{I} \cdot V_{k} \cdot \sin \left(\Phi_{k}+\frac{2 \pi}{\lambda_{0}^{k}}\left[n \cdot x+\left(n-n_{0}\right) \cdot p\right]\right)\right]
$$

where $\mathrm{k}$ labels the interferometric channel corresponding to the point $y_{k}(\mathrm{~A}, \mathrm{~B}, \mathrm{C}$ or $\mathrm{D}), \rho_{k}$ is the trasmission factor, $I_{k}$ the source emission, $\eta_{k}^{P}$ the instrumental visibility factors associated to photometric unbalance, $\eta_{k}^{C}$ and $\eta_{k}^{I}$ the spectral distribution and the wavefront degradation respectively, $V_{k}$ the source visibility and $\Phi_{k}$ the instrument phase. The effective wavelength $\lambda_{0}^{k}$ is slightly different for the four phase channel of each sub-band, in general, but an average value may be defined for each spectral sub-band. This approach does not achieve the desired accuracy, but it can be used in iterative techniques, as a first approximation of the interferometric pattern. A more realistic method, used in the $\mathrm{OPD} / \mathrm{GD}$ algorithm, is to sample the fringe pattern in a small number of point, and use them as first approximation in an iterative least square fit.

\subsection{Algorithm concept}

The evaluation of the OPD modulo lambda allows the identifying of the contribution to the fringe phase due to external factors, i.e. the atmospheric turbulence, while the GD reveals contribution to the interferogram phase over few fringes within the central one, dependent more on astrophysical parameters of the observed source than on atmospheric turbulence. The meaningful difference between OPD modulo lambda and GD is the range, which is one fringe for the OPD and about six fringes for GD. This has physical consequences, i.e. on the kind of noise the two measurements are referred to, but also implementation differences: it means, for GD, more exposure and integration time. This is possible even on faint source because the measurement of the OPD noise is required to close the fringe tracking loop, correcting the current phase value.

The OPD modulo $\lambda$ technique (hereafter called coherent algorithm) is based on a phase demodulation of the combined intensity signals, taking care of the contribution of the instrumental constant phase, supposed as known. The GD estimation (incoherent algorithm) is based on independent phase measurements of the fringe phase in two-three subbands, taking in account the weight of the different wavelengths.

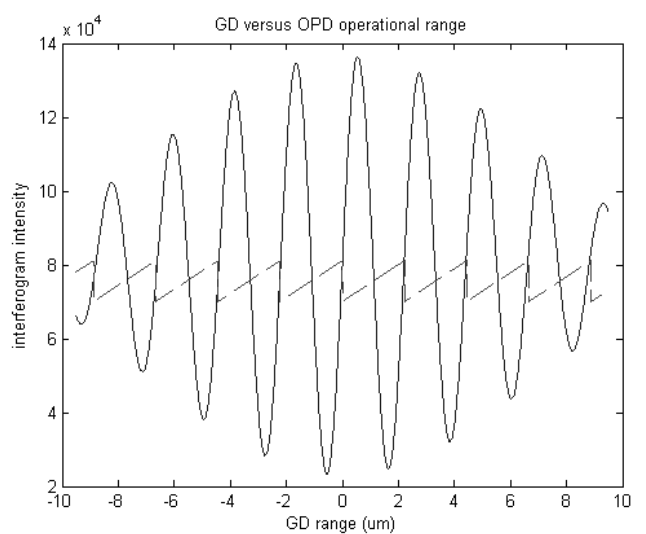

With a careful knowledge on the instrument characteristics and parameters, the current OPD and GD can be evaluated by comparison of the measured interferometric signal with some tabulated FSU output, computed for a set of OPD, respectively GD, and air path values. This allows to identify the most likely OPD or GD value in the least squares sense.

Figure 4 show the relation between the OPD and the GD operational range: the GD measurement operates on a longer range, while the OPD is periodic, as the fringes, by definition (the dashed line)

Fig. 4: OPD modulo $\lambda$ and interferogram profile vs. GD

The celestial source has been modeled as a black body with effective temperatures between $3500 \mathrm{~K}$ and $25000 \mathrm{~K}$; relative fluxes in the three $\mathrm{K}$ sub-bands are scaled accordingly to the current values for the transmission of FSU and for the quantum efficiency of the detector. The instrument model is encoded as a table of optical output values vs. input OPD / GD, with resolution $1 \mathrm{~nm}$, GD range $12.2 \mu \mathrm{m}$, OPD range about $10 \mu \mathrm{m}$, with one fringe length explored at each 
instance. As the OPD is to be stored at a maximum rate of $8 \mathrm{kHz}(200 \mathrm{~Hz}$ for the GD), the total memory occupation for 30 minutes observation is $118.08 \mathrm{MB}$, with a data format of 4 bytes for both OPD/GD and time stamps.

\subsubsection{Coherent algorithm}

The OPD /GD measurement is implemented by an iterative technique which, starting from an initial approximation, generates the new estimate taking advantage of the error gradient descent. The algorithm has been verified to be a rigorous implementation of the Newton-Raphson method for locating the zero of a function, in our case the derivative of the discrepancy between measured data and instrument model.

We define the weighted square discrepancy function: $\quad e(x, z)=\sum_{n}\left(s_{n}(z)-f_{n}(x)\right)^{2} / \sigma_{n}^{2}$,

where $S_{n}(z)$ is the measured FSU output signal corresponding to the current input OPD/GD value $z, f_{n}(x)$ is the modeled FSU output corresponding to a selected OPD/GD value $x$, and $\sigma_{n}^{2}$ is the signal variance; $n$ runs over the FSU channels. The discrepancy is minimised by the best estimate of the input OPD/GD, i.e. $x=z$. It can be derived ${ }^{3}$ that the estimate is unbiased and optimal in the least square sense, when the signal model is correct, and that the RMS error on the estimate is

$$
\sigma^{2}(x)=\left\langle(x-z)^{2}\right\rangle=1 / \sum_{n} \frac{\left[f_{n}^{\prime}\right]^{2}}{\sigma_{n}^{2}}
$$

where $f_{n}^{\prime}$ is the signal derivative. The condition is fulfilled when the error is small with respect to $\lambda / 2 \pi \cong 350 \mathrm{~nm}$. The error function can be estimated by computing the value of the square discrepancy function (eq. 25) over a set of OPD or GD values over the desired range; then the search for the minimum provides the desired least square estimate of the position associated to the current measurement. It is convenient, in the minimum problem, to search the zero of the derivative function versus the $x$ parameter.

The discrepancy derivative function (hereafter, signature) can be written as

$$
h(x, z)=-\sum_{n}\left(s_{n}(z)-f_{n}(x)\right) \cdot f_{n}^{\prime}(x) / \sigma_{n}^{2}=\sum_{n}\left(s_{n}(z)-f_{n}(x)\right) \cdot g_{n}(x)
$$

introducing the weight function

$$
g_{n}(x)=-f_{n}^{\prime}(x) / \sigma_{n}^{2}
$$

The expected values of the signature at the correct location is $h(x, x) \equiv 0$, since it is a minimum.

Factorizing the signal model, we get

$$
h(x, z)=\sum_{n} s_{n}(z) \cdot g_{n}(x)-\sum_{n} f_{n}(x) \cdot g_{n}(x)=\sum_{n} s_{n}(z) \cdot g_{n}(x)-l(x)
$$

where

$$
l(x)=\sum_{n} f_{n}(x) \cdot g_{n}(x)=-\sum_{n} f_{n}(x) \cdot f_{n}^{\prime}(x) / \sigma_{n}^{2}
$$

is a correction term against bias (hereafter, bias).

Starting from a reasonable approximation $x$ of the position estimate, the next approximation $z$ is derived as

$$
z \cong x-\left[\sum_{n} s_{n}(z) \cdot g_{n}(x)-l(x)\right] / h^{\prime}(x, x),
$$

where

$$
h^{\prime}(x, x)=\sum_{n}\left[f_{n}^{\prime}(x)\right]^{2} / \sigma_{n}^{2}
$$


For a new approximation, the procedure of Eq. 31 can be iterated, using the previous estimate as input.

To minimize the computational load, the functions $g, l, 1 / h^{\prime}$ (eq. $28,30,32$ ) can all be tabulated before the start of the observation, given general information on the source and conditions (expected flux, noise, elementary exposure time, air path, environmental conditions), and changed when the above conditions are drastically changed.

\subsubsection{Incoherent algorithm}

The incoherent algorithm for GD requires a global minimum search over the whole GD range; the application of the above method imposes a huge computational load for the need of a large number of reference $x$ positions. A practical solution is to identify the equivalent phase position (i.e. the OPD), corresponding to an estimate within the restricted range of the central fringe period, followed by a verification of the minimum value in corresponding position of the nearby 6 fringes, spanning the desired GD range. The computational load of this implementation is the composition of the OPD basic cycle, plus the computation of the discrepancy (Eq. 10) in a limited set of 6 positions, to identify the global minimum within the 6 discrepancy values. The local value can then be improved by an additional approximation step (eq. 32).

The global minimum search is sensitive to any discrepancy between the real instrument and model parameters, describing both hardware and environmental / observing conditions. Each of them has to be included in the model and updated when necessary, e.g. by auxiliary measurements or calibration.

\subsection{Sensitivity analysis and FSU calibration}

Measurements of OPD and GD requires the whole system to work under known conditions, reasonably close to the nominal configuration. Even small changes in the instrument characteristics from the nominal configuration introduce systematic errors in the OPD/GD; larger variations also degrade the noise performance. Systematic errors are evidenced by the comparison of a nominal signal with the measured signal in a noiseless conditions, perturbing individual parameters, and evaluating the OPD and GD discrepancy. To fulfill the astrometry accuracy requirement of $10 \mu \mathrm{as}$, a maximum discrepancy between the input GD and the measured one (GD bias) of $2.5 \mathrm{~nm}$ over the whole range is required. This parameter can be used as an estimator in the tolerance definition.

\subsubsection{Example: tolerance on fiber misalignment}

As an example, we simulate a misalignment of the four fibers carrying the outputs of the FSU with respect to their nominal position. This reduces their coupling efficiency, causing a loss of flux on selected FSU channels, which may induce an apparent phase shift. Some of the possible displacements can have small impacts, i.e. a displacement in the same direction of all fibers; more complex is the case of shift in different directions.

Figures below show the intensity (in percentage) for transversal and longitudinal (with respect to the spectral dispersion direction) fiber displacements in a range of $10 \mu \mathrm{m}$ centered vs. zero.

The sensitivity analysis shows that transmission variations of order of $1 \%$ can be tolerated with respect to noise performance, and that the uncalibrated systematic error threshold is reached by transmission variations within $\pm 0.5 \%$. A displacement of $\pm 3 \mu \mathrm{m}$ causes a $1 \%$ loss of intensity for the transversal direction, while in longitudinal direction the loss is negligible. In both cases, only the noise performance is considered, since the instrument is assumed to be stable during measurements, so that the current transmission can be included in the calibration.

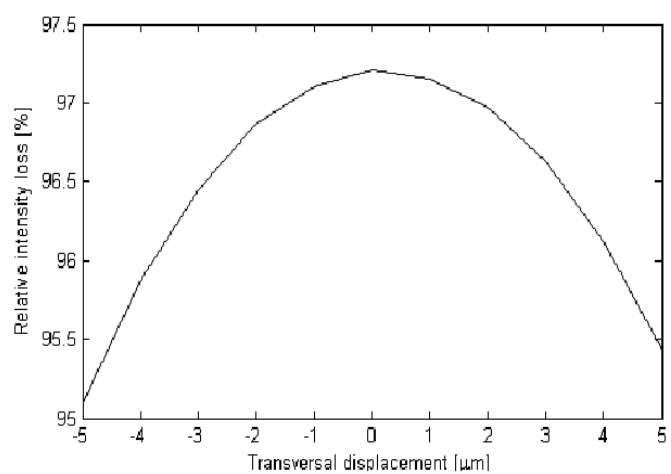

Fig. 5a: Coupled intensity variation vs. transversal displacement

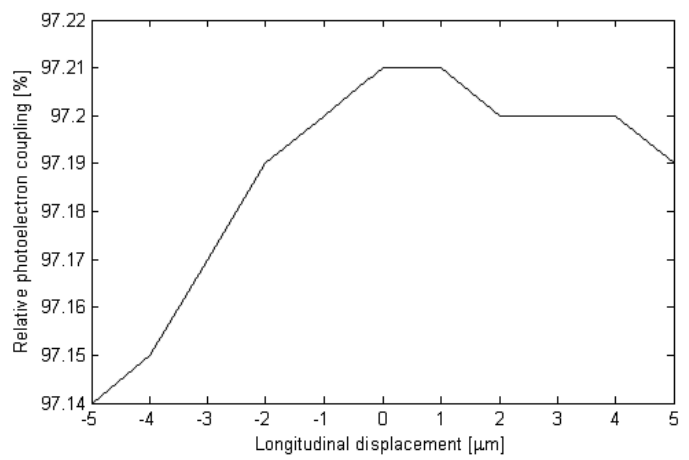

Fig. 5b: Coupled intensity variation vs. longitudinal displacement 


\subsubsection{Impact of pixel response on OPD / GD measurement}

On the detector, the FSU optical performance is modeled by Code V analysis of the optical train; moreover, the on-chip charge diffusion is modeled by a Gaussian, with width $\sigma=8 \mu \mathrm{m}$. The individual pixel response is the superimposition over its area of the contribution at each wavelength of the effective point spread function (PSF). A perturbation of the alignment of fibers modifies the energy distribution on the detector, modifying the intensity on each spectral channel. If we apply a fiber displacement (see section 3.2.1) of $\pm 1 \mu \mathrm{m}$, the spectral response of the three sub-bands is modified as in Fig. 6a: some energy is removed from one side band and sent to the other. In a noiseless signal case, a displacement of $\pm 1 \mu \mathrm{m}$ generate an OPD/GD discrepancy function with opposite slope (Fig. 6b, 6c); at zero input OPD / GD, the discrepancy is also zero, so that the effect is a modification of the apparent measurement effective wavelength, consistent with the displacement of the spectral distribution.

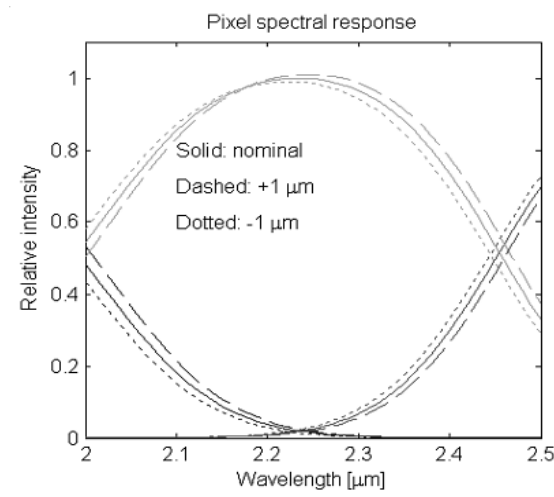

Fig. 6a: spectral response of the three detector channels, nominal (solid line); $+1 \mu \mathrm{m}$ disalignment (dashed line); $-1 \mu \mathrm{m}$ disalignment (dotted line);

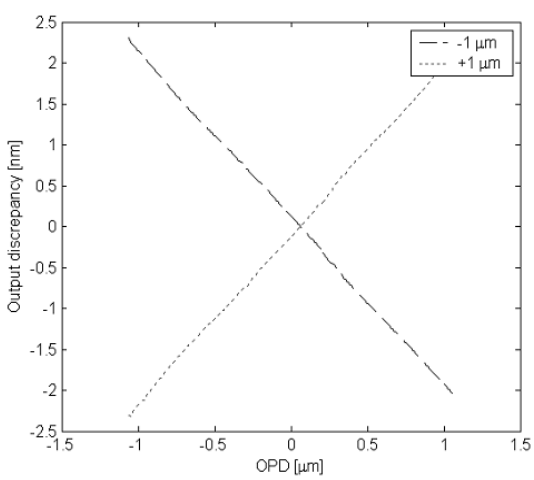

Fig. 6b: OPD output discrepancy vs. input OPD with $+1 \mu \mathrm{m}$ disalignment (dotted line) and $-1 \mu \mathrm{m}$ disalignment (dashed line).

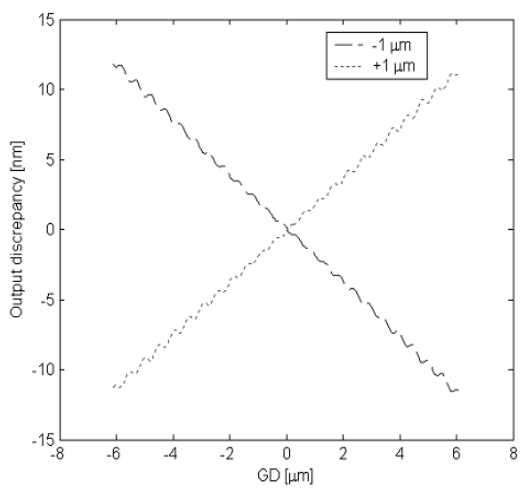

Fig. 6c: GD output discrepancy vs. input GD with $+1 \mu \mathrm{m}$ disalignment (dotted line) and $-1 \mu \mathrm{m}$ disalignment (dashed line).

\subsubsection{Example: FSU effective wavelength}

The FSU signal can be analyzed in order to obtain the instrument spectral response. The Fourier Transform Spectroscopy concept is applied: the FSU signals spectral distribution is given by the modulus of the Fourier transform of the interferogram. A good knowledge of the source characteristic is required. Some constraints are imposed on the observed object, modeled as a black body with effective temperature in a range of [3500-25000] K. Limits of the temperature error variance for the sky object is deduced by constraint on related parameters. We choose, as an example, the measured effective wavelength. This is provided by composition of the source spectrum (which may define the object effective wavelength), the transmission of the atmosphere and of the VLTI transfer optics, and eventually of the FSU transmission and detector spectral response.

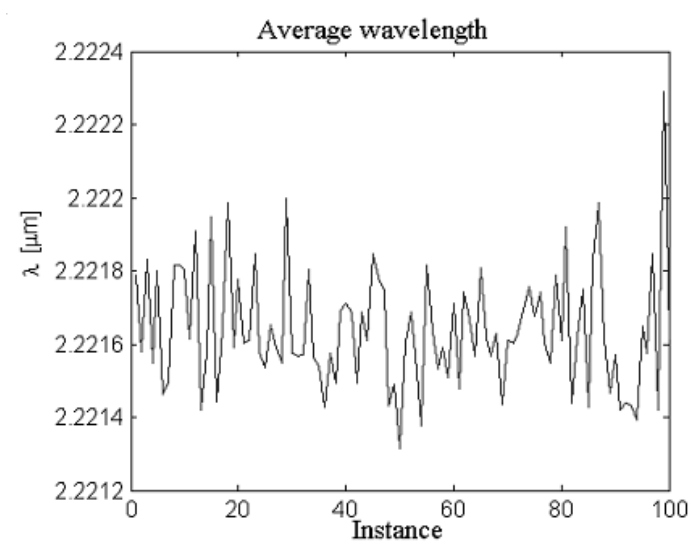

Fig. 7: fluctuations of the estimated effective wavelength in a Monte Carlo of cases affected by realistic noise
Assuming the source is reasonably well known, it is possible with a single global measurement to assess the overall response of the complete measurement chain, providing an update to the FSU model consistent with the current observing conditions. This figure show a Monte Carlo simulation over 100 instances in a noisy situation, with a background noise of $1 \mathrm{e} 5$ photons/s, source magnitude $\mathrm{K}=10 \mathrm{mag}$, nominal FSU configuration.
MEW mean
$2.22164 \mathrm{~mm}$
MEW standard dev:
$0.164 \mathrm{~nm}$ 
To fulfill the requirement on the effective wavelength measurement, the source effective temperature must be known to a precision ranging from few ten degrees for red stars $(\mathrm{T}=3500 \mathrm{~K})$ to $2000 \mathrm{~K}$ for blue stars $(\mathrm{T}=25000 \mathrm{~K})$, corresponding to a $\mathrm{K}$ band brightness stability ranging from $0.02 \mathrm{mag}$ to $0.1 \mathrm{mag}$, respectively.

\section{CONCLUSIONS}

Both FINITO and PRIMA FSU can be described by similar models of polychromatic interferogram, including the description of instrument transmission, phase and visibility. In this initial phase of interferometer optimisation, a number of algorithms have been considered, for OPD and GD measurement, for internal metrology monitoring, etc.

Currently, the fringe tracking loop is routinely closed on siderostats, and future operation on UT and AT may take advantage of a range of algorithms optimised for the specific observing conditions.

A key relevance will be associated to instrument parameters monitoring and calibration, which can be performed in a global strategy.

\section{REFERENCES}

1. A. Glindemann, R. Abuter, F. Carbognani, F. Delplancke, F. Derie, A. Gennai, P. B. Gitton, P. Kervella, B. Koehler, S. A. Lévêque, S. Menardi, A. Michel, F. Paresce, T. P. Duc, A. Richichi, M. Schõller, M. Tarenghi, A. Wallander, R. Wilhelm: VLT interferometer: a unique instrument for high-resolution astronomy, Proc. SPIE 4006-2, 2000.

2. M. Gai, S. Menardi, S. Cesare, B. Bauvir, D. Bonino, L. Corcione, M. Dimmler, G. Massone, F. Reynaud, A. Wallander: The VLTI Fringe Sensor Units: FINITO and PRIMA FSU, Proc. SPIE 5491-61 (these conferences), 2004

3. M. Gai, S. Casertano, D. Carollo, M. G. Lattanzi: Location estimators for interferometric fringes, PASP 110, 848862, 1998.

4. P. R. Lawson, M. M. Colavita, P. J. Dumont, B. F. Lane: Least-Square Estimation and Group Delay in Astrometric Interferometers, Proc. SPIE 4006-46, 2000 